01

\title{
Тепловое излучение абсолютно черного тела, движущегося в равновесном газе фотонов
}

\author{
(ㄷ Г.В. Дедков, А.А. Кясов \\ Кабардино-Балкарский государственный университет, \\ 360004 Нальчик, Россия \\ e-mail: gv_dedkov@mail.ru
}

Поступило в Редакцию 14 декабря 2020 г.

В окончательной редакции 15 января 2021 г.

Принято к публикации 28 января 2021 г.

Рассмотрены динамика, кинетика теплообмена и интенсивность теплового излучения абсолютно черного тела с собственной температурой $T_{1}$, движущегося с произвольной скоростью в равновесном газе фотонов с собственной температурой $T_{2}$, не зависящей от времени. Получены формулы для спектрально-угловой и полной интенсивности излучения, а также других величин в системе покоя тела и в системе отсчета фотонного газа. Показано, что в начальный момент интенсивность излучения сферических и дискообразных частиц одинакового радиуса по-разному зависит от скорости движения и соотношения температур $T_{1}$ и $T_{2}$. Затем устанавливается квазистационарное тепловое состояние тел с эффективной температурой, зависящей от скорости и температуры $T_{2}$, интенсивность теплового излучения не зависит от формы, а кинетическая энергия трансформируется в излучение. Характерное время установления квазистационарного состояния на много порядков величины меньше характерного времени торможения.

Ключевые слова: тепловое излучение абсолютно-черного движущегося тела.

DOI: $10.21883 /$ JTF.2021.07.50947.343-20

\section{Введение}

Тепловое излучение тел с произвольной геометрией и материальными свойствами имеет фундаментальное значение для физики, астрофизики и микротехнологии. В случае движущихся тел оно имеет свои особенности. В настоящее время интерес к этому вопросу стимулируется развитием микроэлектромеханических (МЭМС) и микрооптомеханических (МОМС) систем [1-3], исследованиями быстро вращающихся частиц в атомных ловушках $[4,5]$ и астрофизическими приложениями [6-10].

Однако, как ни странно, даже вопрос о тепловом излучении абсолютно черных движущихся тел (АЧТ) изучен недостаточно полно. Так, тело, имеющее собственную температуру $T_{1}$, заданную в собственной системе покоя $\Sigma^{\prime}$, движущееся со скоростью $V$ в равновесном вакуумном фоне электромагнитного излучения (фотонном газе) с температурой $T_{2}$, заданной в системе покоя $\Sigma$ фотонного газа, излучает тепловые фотоны, теряя энергию. В то же время оно нагревается и тормозится фоновым излучением, поскольку импульс „встречных“ фотонов больше импульса ,догоняющих“ из-за эффекта Доплера. Не умаляя общности, будем считать, что система отсчета $\Sigma^{\prime}$ движется вместе с телом вдоль оси $x$ системы отсчета $\Sigma$, совпадающей с направлением оси $x^{\prime}$ в $\Sigma^{\prime}$ (рис. 1).

Очевидно, что в рассматриваемом случае процессы излучения и поглощения должны зависеть от скорости тела, его геометрических и материальных параметров. Целью настоящей работы является расчет физических величин, характеризующих интенсивность излучения движущегося АЧТ, размер которого превышает виновскую длину волны $\left(R \gg \lambda_{W}\right)$. Рассматриваются кинетика нагрева и динамика торможения тела, спектральная и полная интенсивности излучения, роль формы и других параметров. Противоположный $\left(R \ll \lambda_{W}\right)$ случай рассматривался в [11], причем движение субмикронных частиц (не абсолютно-черных, с заданными диэлектрическими свойствами) предполагалось поступательно-вращательным. Для более крупных тел, рассматриваемых в настоящей работе, вращательные эффекты (в первом приближении) можно не учитывать.

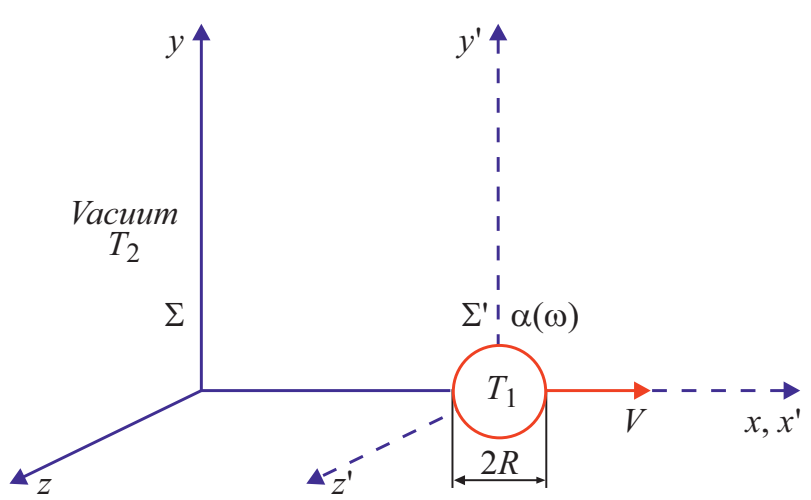

Рис. 1. Система отсчета фотонного газа $\Sigma$ и система покоя $\Sigma^{\prime}$ движущегося тела. 


\section{1. Теоретические соотношения}

При указанных выше условиях для любого тела, движущегося относительно равновесного фотонного газа, выполняется несколько общих соотношений, связывающих физические величины, характеризующие его тепловой нагрев, излучение и торможение [10-14]:

$$
\begin{gathered}
d Q^{\prime} / d t^{\prime}=-\left(I_{1}^{\prime}-I_{2}^{\prime}\right)=\gamma^{2} d Q / d t, \\
I=I_{1}-I_{2}=-\left(d Q / d t+F_{x} V\right), \\
F_{x}^{\prime}=F_{x}-\left(\beta \gamma^{2} / c\right) d Q / d t .
\end{gathered}
$$

Штрихованные и нештрихованные величины в (1)-(3) и далее соответствуют системам отсчета $\Sigma^{\prime}$ и $\Sigma$. В частности, $I_{1}^{\prime}$ и $I_{2}^{\prime}$ обозначают интегральные интенсивности собственного излучения тела и поглощаемого фонового излучения в системе отсчета $\Sigma$ фотонного газа, а $I_{1}^{\prime}$ и $I_{2}^{\prime}$ - в системе покоя тела $\Sigma^{\prime} ; F_{x}$ и $F_{x}^{\prime}-$ тангенциальные силы, действующие на тело, $\beta=F / c$, $\gamma=\left(1-\beta^{2}\right)^{-1 / 2}$. Целесообразность введения отдельных слагающих интенсивности обусловлена тем, что они зависят от разных температур и, кроме того, $I_{1}$ и $I_{1}^{\prime}$ могут включать нетепловое излучение движущегося тела, как, например, при поступательно-вращательном движении [11]. Отметим также, что использование собственных температур тела и фотонного газа избавляет от необходимости рассмотрения трансформационных свойств температуры в различных инерциальных системах отсчета. Обсудим подробнее смысл величин, входящих в (1)-(3).

В рамках флуктуационной электродинамики, адекватно описывающей процессы теплового излучения и поглощения нагретых тел с произвольными материальными свойствами, производные $d Q^{\prime} / d t$ и $d Q / d t$ определяются соотношениями [12]

$$
\begin{gathered}
\frac{d Q^{\prime}}{d t^{\prime}}=\int\left\langle\mathbf{j}^{\prime} \mathbf{E}^{\prime}\right\rangle d^{3} r^{\prime}=\int\left\langle\frac{\partial \mathbf{P}^{\prime}}{\partial t^{\prime}}+\frac{\partial \mathbf{M}^{\prime}}{\partial t^{\prime}} \mathbf{B}\right\rangle d^{3} r^{\prime}, \\
\int\langle\mathbf{j} \mathbf{E}\rangle d^{3} r \equiv F_{x} V+d Q / d t,
\end{gathered}
$$

где интегралы берутся по объему тела, а угловые скобки (здесь и далее) означают полное квантовостатистическое усреднение. В выражениях (4), (5) j и $\mathbf{j}^{\prime}$ - плотности тока внутри тела, связанные с плотностями заряда $\rho\left(\rho^{\prime}\right)$, векторами поляризации $\mathbf{P}\left(\mathbf{P}^{\prime}\right)$ и намагниченности $\mathbf{M}\left(\mathbf{M}^{\prime}\right)$ стандартными соотношениями $\mathbf{j}=\partial \mathbf{P} / \partial t+c \operatorname{rot} \mathbf{M}, \rho=-\operatorname{div} \mathbf{P}$ (и аналогично для величин в $\left.\Sigma^{\prime}\right), \mathbf{E}\left(\mathbf{E}^{\prime}\right)$ и $\mathbf{B}\left(\mathbf{B}^{\prime}\right)$ - векторы электрического и магнитного полей в $\Sigma$ и $\Sigma^{\prime}$. Выполняя релятивистские преобразования переменных в интеграле (4), получим выражение, эквивалентное (1) [12]:

$$
\int\left\langle\frac{\partial \mathbf{P}^{\prime}}{\partial t^{\prime}} \mathbf{E}^{\prime}\right\rangle d^{3} r^{\prime}=\gamma^{2} \int\left\langle\frac{\partial \mathbf{P}}{\partial t} \mathbf{E}+\frac{\partial \mathbf{M}}{\partial t} \mathbf{B}\right\rangle d^{3} r .
$$

Левая часть (6), в силу определения (4), описывает скорость нагрева тела в собственной системе отсчета $\Sigma^{\prime}$.
В отличие от этого величина $d Q / d t$, представленная интегралом в правой части (6) без гамма-фактора и входящая в (5), имеет самостоятельное значение и совпадает со скоростью нагрева тела в системе отсчета $\Sigma$ только при нерелятивистском движении. В случае малой частицы $\left(R \ll \lambda_{W}\right)$ с флуктуационными дипольными электрическим и магнитным дипольными моментами d и $\mathbf{m}$ формула (6) преобразуется к более простому виду [9]

$$
\left\langle\dot{\mathbf{d}}^{\prime} \mathbf{E}^{\prime}+\dot{\mathbf{m}} \mathbf{B}^{\prime}\right\rangle=\gamma^{2}\langle\mathbf{d} \mathbf{E}+\dot{\mathbf{m}} \mathbf{B}\rangle,
$$

где точки над векторами $\mathbf{d}$ и $\mathbf{m}$ обозначают производные по времени.

Как легко видеть, тождество (2) вытекает из (5) и (8) при условии квазистационарности $d W / d t=0$, являясь следствием закона сохранения энергии системы „движущееся тело-электромагнитное поле“ [13]:

$$
-d W / d t=\oint_{\sigma} S_{n} \cdot d \sigma+\int_{\Omega}\langle\mathbf{j} \cdot \mathbf{E}\rangle d^{3} r .
$$

Здесь $W$ - энергия электромагнитного поля, $S_{n}$ - нормальная проекция вектора Пойнтинга $\mathbf{S}=(c / 4 \pi)\langle E \times \mathbf{H}\rangle$ на волновую поверхность $\sigma$, окружающую тело, $\Omega$ - объем тела. Из (2), в частности, следует, что при $d Q / d t=0$ движущееся тело излучает за счет прямого превращения кинетической энергии в тепловое излучение. В общем же случае интенсивность излучения зависит от кинетики теплового нагрева (охлаждения) тела.

В свою очередь, тождество (3) является следствием преобразования Лоренца для импульса тела (при переходе от $\Sigma^{\prime}$ к $\Sigma$ ) и уравнения динамики, учитывающего изменение массы при излучении: $d Q^{\prime} / d t^{\prime}=\left(d m / d t^{\prime}\right) c^{2}$. Эффект изменения массы особенно важен для динамики микрочастиц.

Простейший путь вычисления интенсивностей $I_{1}, I_{2}$ состоит в нахождении величин $F_{x}^{\prime}$ и $d Q^{\prime} / d t^{\prime}=\left(d m / d t^{\prime}\right) c^{2}$, а затем $F_{x}$ и $I_{1}, I_{2}$ с помощью выражений (1)-(3). При этом сила $F_{x}^{\prime}$ зависит только от температуры $T_{2}$ фотонного газа, поскольку собственное тепловое излучение тела не приводит к появлению реактивного импульса. Необходимые для расчета величины плотности энергии $\varepsilon^{\prime}$ и потока импульса $S_{x}^{\prime} / c$ фонового электромагнитного излучения в системе отсчета $\Sigma^{\prime}$ являются компонентами тензора энергии-импульса $T^{\prime \mu \nu}$ и выражаются через компоненты $T^{\alpha \beta}$ этого тензора в системе отсчета $\Sigma$ с помощью преобразования Лоренца $\Lambda_{\alpha}^{\mu}: T^{\prime \mu \nu}=\Lambda_{\alpha}^{\mu} \Lambda_{\beta}^{\nu} T^{\alpha \beta}$, которое удобно записать в матричной форме

$$
T^{\prime}=\Lambda T \Lambda^{T}=\Lambda T \Lambda \text {. }
$$

В системе отсчета $\Sigma$ матрица тензора $T^{\alpha \beta}$ имеет вид $[15]$

$$
T=\left(\begin{array}{llll}
\varepsilon & 0 & 0 & 0 \\
0 & p & 0 & 0 \\
0 & 0 & p & 0 \\
0 & 0 & 0 & p
\end{array}\right),
$$

где $p=\varepsilon / 3, \quad \varepsilon=4 \sigma_{B} T_{2}^{4} / c, \quad \sigma_{B}-$ постоянная Стефана-Больцмана. В свою очередь, матрица Лоренца 
имеет вид

$$
\Lambda=\left(\begin{array}{cccc}
\gamma & -\gamma \beta & 0 & 0 \\
-\gamma \beta & \gamma & 0 & 0 \\
0 & 0 & 1 & 0 \\
0 & 0 & 0 & 1
\end{array}\right)
$$

Подставляя (10), (11) в (9), получим

$$
T^{\prime \mu \nu}=\varepsilon \cdot\left(\begin{array}{cccc}
\gamma^{2}\left(1+\beta^{2} / 3\right) & -4 \gamma^{2} \beta / 3 & 0 & 0 \\
-4 \gamma^{2} \beta / 3 & \gamma^{2}\left(\beta^{2}+1 / 3\right) & 0 & 0 \\
0 & 0 & 1 / 3 & 0 \\
0 & 0 & 0 & 1 / 3
\end{array}\right) \text {, }
$$

Из (12) следует, что в соответствии со смыслом компонент тензора энергии-импульса [15] плотность энергии и плотность потока импульса фонового теплового излучения в собственной системе отсчета тела $\Sigma^{\prime}$ определяются выражениями

$$
\begin{gathered}
\varepsilon^{\prime}=T^{\prime 00}=\gamma^{2}\left(1+\beta^{2} / 3\right) \varepsilon=(4 / c) \sigma_{B} T_{2}^{4} \gamma^{2}\left(1+\beta^{2} / 3\right), \\
S_{x}^{\prime} / c=T^{\prime 01}=T^{\prime 10}=-4 \gamma^{2} \beta \varepsilon / 3=-(16 / 3) \gamma^{2} \beta \sigma_{B} T_{2}^{4} / c .
\end{gathered}
$$

Сила, действующая на сферическое тело радиуса $R$ или тонкий диск радиуса $R$ (если плоскость диска перпендикулярна вектору скорости), равна

$$
\begin{aligned}
F_{x}^{\prime} & =\sigma_{a b s} S_{x}^{\prime} / c=-(16 / 3) \sigma_{B} \pi R^{2} \beta \gamma^{2} T_{2}^{4} / c \\
& =-(4 / 3) \beta \gamma^{2} a T_{2}^{4} / c,
\end{aligned}
$$

где $\sigma_{a b s}=\pi R^{2}-$ сечение поглощения (как для сферы, так и для диска), $a=4 \pi R^{2} \sigma_{B}$. Интересно, что формула (15) в отличие от приведенных далее выражений для интенсивности излучения и других величин была впервые получена еще полвека назад [16]. Интенсивность излучения и скорость нагрева тела $d Q^{\prime} / d t^{\prime}$ в системе отсчета $\Sigma^{\prime}$ легко найти, учитывая (13) и левую часть (1) в форме $d Q^{\prime} / d t^{\prime}=-I^{\prime}=-\left(I_{1}^{\prime}-I_{2}^{\prime}\right)$. В частности, для диска будем иметь

$$
\begin{gathered}
I_{1}^{\prime}=2 \pi R^{2} \sigma_{B} T_{1}^{4}=a T_{1}^{4} / 2, \\
I_{2}^{\prime}=(c / 4) \varepsilon^{\prime} 2 \pi R^{2}=a T_{2}^{4} \gamma^{2}\left(1+\beta^{2} / 3\right) / 2,
\end{gathered}
$$

поэтому

$$
\begin{gathered}
I^{\prime}=I_{1}^{\prime}-I_{2}^{\prime}=a T_{1}^{4} / 2-a T_{2}^{4} \gamma^{2}\left(1+\beta^{2} / 3\right) / 2, \\
d Q^{\prime} / d t^{\prime}=-a T_{1}^{4} / 2+a T_{2}^{4} \gamma^{2}\left(1+\beta^{2} / 3\right) / 2 .
\end{gathered}
$$

Для сферы площадь поверхности вдвое больше, поэтому формулы, аналогичные (16) и (17), имеют вид

$$
I^{\prime}=4 \pi R^{2}\left(\sigma_{B} T_{1}^{4}-(c / 4) \varepsilon^{\prime}\right)=a T_{1}^{4}-a T_{2}^{4} \gamma^{2}\left(1+\beta^{2} / 3\right),
$$

$$
d Q^{\prime} / d t^{\prime}=-a T_{1}^{4}+a T_{2}^{4} \gamma^{2}\left(1+\beta^{2} / 3\right) .
$$

Дальнейшее элементарное вычисление интенсивностей $I_{1}$ и $I_{2}$ с учетом формул (1)-(3), (16)-(19) дает: а) диск

$$
I=a\left(T_{1}^{4}-T_{2}^{4}\right) / 2+(2 / 3) a \beta^{2} \gamma^{2} T_{2}^{4}
$$

б) сфера

$$
I=a\left(T_{1}^{4}-T_{2}^{4}\right) .
$$

Из (1), (3), (17) и (19) вытекают также выражения для тангенциальной силы $F_{x}$ и производной $\dot{Q}$, заданных в системе отсчета $\Sigma$ :

а) диск

$$
\begin{gathered}
F_{x}=-\frac{a \beta}{2 c}\left[T_{1}^{4}+\frac{\left(5-\beta^{2}\right)}{3} \gamma^{2} T_{2}^{4}\right], \\
\dot{Q}=-\frac{a}{2} \gamma^{-2}\left[T_{1}^{4}-\left(1+\beta^{2} / 3\right) \gamma^{2} T_{2}^{4}\right] ;
\end{gathered}
$$

б) сфера

$$
\begin{gathered}
F_{x}=-\frac{a \beta}{c}\left[T_{1}^{4}+T_{2}^{4} / 3\right], \\
\dot{Q}=-a \gamma^{-2}\left\lfloor T_{1}^{4}-\left(1+\beta^{2} / 3\right) \gamma^{2} T_{2}^{4}\right\rfloor .
\end{gathered}
$$

Из выражений (20), (21) следует, что при фиксированных температурах $T_{1}$ и $T_{2}$ в начальный момент времени тепловое излучение сферического тела не зависит от скорости, в отличие от излучения диска.

\section{2. Кинетика теплообмена, динамика торможения и квазистационарное излучение}

Предположим, что температура $T_{2}$ фотонного газа не изменяется с течением времени. Тогда из (18), (19) следует, что в результате теплообмена с ним тело достигает состояния квазистационарного равновесия, при котором его собственная эффективная температура определяется условием $d Q^{\prime} / d t^{\prime}=-I^{\prime}=0$. Приравнивая к нулю правую часть (19), будем иметь

$$
T_{1}^{(e)}=T_{2} \gamma^{1 / 2}\left(1+\beta^{2} / 3\right)^{1 / 4},
$$

причем величина $T_{1}^{(e)}$ одинакова и для диска, и для сферы. Именно такая величина квазиравновесной температуры была получена другим методом авторами задачника [17] (задача 5.12). Подставляя (26) в (20) и (21), получим одинаковую результирующую интенсивность квазистационарного излучения и для сферы, и для диска:

$$
I^{(e)}=(4 / 3) a T_{2}^{4} \gamma^{2} \beta^{2} .
$$

В соответствии с (1), (2) в этом режиме происходит прямое превращение кинетической энергии тела в излучение без изменения его теплового состояния (и состояния фотонного газа). Однако до наступления 
этого режима, как следует из (20), (21), интенсивности излучения сферы и диска могут заметно отличаться. Поскольку $T_{1}^{(e)}$ и $I^{(e)}$ зависят от скорости частицы, динамику торможения и кинетику теплообмена необходимо рассмотреть более детально. Уравнение динамики имеет вид

$$
\frac{d}{d t} \frac{m V}{\sqrt{1-\beta^{2}}}=F_{x} .
$$

С учетом скорости изменения массы за счет нагрева тела $\left.\left(d Q^{\prime} / d t^{\prime}\right)=d m / d t^{\prime}\right) c^{2}$, релятивистского замедления времени $d t^{\prime}=d t / \gamma$ и тождества (1) выражение для силы $F_{x}$ принимает вид [14]

$$
F_{x}=F_{x}^{\prime}+\gamma V d m / d t
$$

а уравнение (29) соответственно

$$
m \frac{d V}{d t}=\gamma^{-3 / 2} F_{x}^{\prime}
$$

Подставляя (15) в (30), приходим к уравнению

$$
\frac{d \beta}{\beta \sqrt{1-\beta^{2}}}=-\frac{4 a T_{2}^{4}}{3 m c^{2}} d t .
$$

Интегрируя (31), получим

$$
\beta(t)=\frac{2 \beta_{0} \exp \left(-8 a T_{2}^{4} t / 3 m c^{2}\right)}{2-\beta_{0}\left(1-\exp \left(-8 a T_{2}^{4} t / 3 m c^{2}\right)\right)},
$$

где $\beta_{0}=\beta(0)$. Из (32) следует, что характерное время торможения тела равно

$$
t_{\beta}=\frac{3 m c^{2}}{8 a T_{2}^{4}}=\frac{R \rho c^{2}}{8 \sigma_{B} T_{2}^{4}}
$$

где $\rho$ - плотность тела (в данном случае имеем в виду сферическое тело). Например, для шарика льда $\left(\mathrm{H}_{2} \mathrm{O}\right)$ с радиусом $R=1 \mathrm{~cm}$ и с плотностью $\rho=0.9 \mathrm{~g} / \mathrm{cm}^{3}$ при температуре $T_{2}=50 \mathrm{~K}$ (эти условия характерны для космических облаков газа и пыли в протозвездных конденсациях) время замедления составит $2.7 \cdot 10^{10}$ лет. T. е. если при таких условиях шарик получил начальный импульс, то в течение всего времени эволюции облака $\left(\sim 10^{7}\right.$ лет) его скорость уменьшается незначительно (при отсутствии столкновений и нагрева прямым излучением протозвезд). В то же время его тепловое состояние, как показано ниже, изменяется значительно быстрее.

Для анализа кинетики теплового нагрева (охлаждения) тела используем уравнение (19), левую часть которого запишем в виде $\left(C_{s}-\right.$ удельная теплоемкость тела)

$$
\frac{d Q}{d t^{\prime}}=\frac{d}{d t^{\prime}}\left(C_{s} m T_{1}\right)=\gamma C_{s} m \frac{d T_{1}}{d t}+\gamma C_{s} T_{1} \frac{d m}{d t}+\gamma m T_{1} \frac{d C_{s}}{d t} .
$$

С учетом (1) и соотношения $d Q^{\prime} / d t^{\prime}=\left(d m / d t^{\prime}\right) c^{2}$ уравнение (34) принимает вид

$$
C_{s} m \frac{d T_{1}}{d t}=\gamma^{-1} \frac{d Q^{\prime}}{d t^{\prime}}\left(1-C_{s} T_{1} / c^{2}\right)-m T_{1} \frac{d C_{s}}{d t} .
$$

При температурах существования твердых тел независимо от их природы $C_{s} T_{1} / c^{2} \ll 1$ (см. далее), поэтому этой величиной можно пренебречь, а для зависимости теплоемкости тела от температуры (при $T>T_{0}$ ) используем линейную аппроксимацию $C_{s}(T)=C_{0}\left(1+b\left(T-T_{0}\right)\right)$. Далее, подставляя (19) в (35) и делая замену переменных $T_{1}=x T_{2}, t=\tau t_{Q}$, $t_{Q}=\frac{\gamma R C_{0} \rho}{3 \sigma_{B} T_{2}^{3}}$, уравнение (35) приводится к виду (здесь также имеем в виду сферическое тело)

$$
\frac{d x}{d \tau}(A+B x)=-x^{4}+\gamma^{2}\left(1+\beta^{2} / 3\right),
$$

где $A=1-b T_{0}, B=b T_{2}$.

Интегрируя (36) с учетом начального условия $x(0)=x_{0}$, получим

$$
\ln \left(f(x) / f\left(x_{0}\right)\right)=2 \alpha^{3} \tau+\operatorname{arctg}\left(x_{0} / \alpha\right)-\operatorname{arctg}(x / \alpha),
$$

где параметр $\alpha=\gamma^{1 / 2}\left(1+\beta^{2} / 3\right)^{1 / 4}$ определяет квазистационарную температуру тела (см. (26)), а

$$
f(x)=\left(\frac{\alpha^{2}+x^{2}}{\alpha^{2}+x_{0}^{2}}\right)^{B} \frac{(\alpha+x)^{0.5 A-B}}{|\alpha-x|^{0.5 A+B}} .
$$

Из (37) следует, что условие $\tau \rightarrow \infty$ выполняется при $x \rightarrow \alpha$, поэтому с учетом асимптотики $f(x)$ при $x \rightarrow \alpha$ для характерного времени установления квазистационарной температуры будем иметь

$$
t^{(e)} \approx t_{Q}(0.5 A+B) / 2 \alpha^{3}=\frac{R C_{0} \rho(0.5 A+B)}{6 \gamma^{1 / 2}\left(1+\beta^{2} / 3\right)^{3 / 4} \sigma_{B} T_{2}^{3}} .
$$

Учитывая (33) и (38), для отношения времен $t^{(e)} / t_{\beta}$ находим

$$
t^{(e)} / t_{\beta}=\frac{4 C_{0} T_{2}}{3 c^{2}} \gamma^{-1 / 2}\left(1+\beta^{2} / 3\right)^{-3 / 4}(0.5 A+B) .
$$

В интервале температур 10-300 K, при типичных значениях параметров для многих твердых соединений: $C_{0}=1-10 \mathrm{~J} / \mathrm{K}, T_{0}=1-10 \mathrm{~K}, b=0.1-0.2 \mathrm{~K}^{-1}[18]$, полагая $\gamma \approx 1$ и $T_{2}=50 \mathrm{~K}$, получим $t^{(e)} / t_{\beta} \approx 10^{-14}$, так что время установления квазистационарного состояния, действительно, гораздо меньше времени торможения, оправдывая приближение, использованное при решении уравнения (35).

\section{3. Спектр излучения}

Согласно [19], спектрально-угловая излучательная способность $1 \mathrm{~cm}^{2}$ поверхности АЧТ с заданной температурой $T_{1}$ (в системе отсчета $\Sigma^{\prime}$ в нашем случае) 


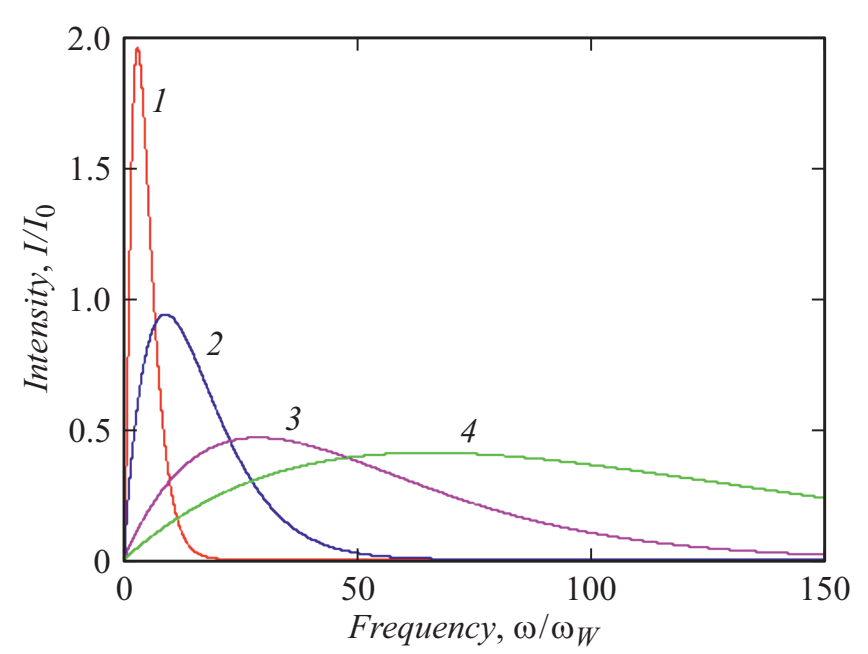

Рис. 2. Спектр излучения движущегося тела в системе отсчета фотонного газа. Кривые $1-4$ соответствуют $\beta=0.5,0.95$, $0.995,0.999$. Кривые $2-4$ показаны с увеличением в 3,5 и 10 pas, $I_{0}=\hbar \omega_{W}^{3} \sigma_{a b s} /\left(2 \pi^{2} c^{2}\right)$.

определяется выражением

$$
\begin{aligned}
& J^{\prime}\left(\omega^{\prime}, \vartheta^{\prime}, \varphi^{\prime}\right) d \omega^{\prime} d \Omega^{\prime} \\
& =\frac{\hbar \omega^{\prime 3}}{4 \pi^{3} c^{2}} \cos \vartheta\left[\exp \left(\hbar \omega^{\prime} / k_{B} T_{1}\right)-1\right]^{-1} d \omega^{\prime} d \Omega^{\prime} .
\end{aligned}
$$

В этом выражении $\widehat{\vartheta}$ - угол между направлением излучения и вектором нормали к элементу поверхности тела, а углы $\vartheta^{\prime} \varphi^{\prime}$ сферической координатной системы определим таким образом, что $\vartheta^{\prime}$ отсчитывается от направления оси $x^{\prime}$, совпадающей с направлением вектора скорости тела. Телесный угол $d \Omega^{\prime}$ соответствует направлению излучения. Суммируя излучение от всех элементов поверхности, получим

$$
\begin{aligned}
& I^{\prime}\left(\omega^{\prime}, \vartheta^{\prime}, \varphi^{\prime}\right) d \omega^{\prime} d \Omega^{\prime}=\frac{\hbar \omega^{\prime 3}}{4 \pi^{3} c^{2}} \\
& \times \sigma_{a b s}\left[\exp \left(\hbar \omega^{\prime} / k_{B} T_{1}\right)-1\right]^{-1} d \omega^{\prime} d \Omega^{\prime},
\end{aligned}
$$

где $I^{\prime}\left(\omega^{\prime}, \vartheta^{\prime}, \varphi^{\prime}\right)$ - спектрально-угловая интенсивность излучения, определяемая также выражением (здесь $E^{\prime}-$ энергия тела)

$$
\frac{d^{3} E^{\prime}}{d t^{\prime} d \omega^{\prime} d \Omega^{\prime}}=I^{\prime}\left(\omega^{\prime}, \vartheta^{\prime}, \varphi^{\prime}\right),
$$

a сечение поглощения $\sigma_{a b s}$ равно максимальной площади сечения тела плоскостью, перпендикулярной к направлению вектора излучения. Для сферического тела и для диска радиуса $R$, очевидно, $\sigma_{a b s}=\pi R^{2}$. Учитывая релятивистские преобразования энергии, частоты, времени и телесного угла при переходе от $\Sigma^{\prime}$ к $\Sigma$, левая часть (42) принимает вид

$$
\frac{d^{3} E^{\prime}}{d t^{\prime} d \omega^{\prime} d \Omega^{\prime}}=\frac{d^{3} E}{d t d \omega d \Omega} \gamma^{3}(1-\beta \cos \vartheta)^{2},
$$

а выражение (39) для $I\left(\omega^{\prime}, \vartheta^{\prime} \varphi^{\prime}\right)$ соответственно

$$
\begin{aligned}
& I\left(\omega^{\prime}, \vartheta^{\prime}, \varphi^{\prime}\right)=\frac{\hbar \omega^{3}}{4 \pi^{3} c^{2}} \sigma_{a b s} \gamma^{3}(1-\beta \cos \vartheta)^{3} \\
& \times\left[\exp \left(\hbar \gamma \omega(1-\beta \cos \vartheta) / k_{\beta T_{1}}\right)-1\right]^{-1}
\end{aligned}
$$

Из (43), (44) следует, что спектрально-угловая интенсивность излучения тела в системе отсчета $\Sigma$ равна

$$
\begin{aligned}
\frac{d^{3} E}{d t d \omega d \Omega} & =I(\omega, \vartheta, \varphi)=\frac{\hbar \omega^{3}}{4 \pi^{3} c^{2}} \sigma_{a b s}(1-\beta \cos \vartheta) \\
& \times\left[\exp \left(\hbar \gamma \omega(1-\beta \cos \vartheta) / k_{\beta} T_{1}\right)-1\right]^{-1}
\end{aligned}
$$

Формула (45) согласуется с [16]. При условии квазистационарного равновесия тела с фоновым излучением температура $T_{1}$ в (43) определяется формулой (26). Интегрируя (45) по частотам, получим угловое распределение излучения

$$
I(\vartheta, \varphi)=\frac{\sigma_{B} T_{1}^{4}}{\pi} \sigma_{a b s} \gamma^{-4}(1-\beta \cos \vartheta)^{-3},
$$

а интегрируя (45) по телесному углу $d \Omega=2 \pi \sin \vartheta d \vartheta-$ спектральное распределение

$$
\begin{aligned}
I(\omega) & =\frac{\hbar}{2 \pi^{2} c^{3}} \sigma_{a b s} \gamma^{-2} \beta^{-1} \omega_{W}^{2} \omega \\
& \times\left[f\left(\frac{\omega}{\omega_{W}} \sqrt{\frac{1-\beta}{1+\beta}}\right)-f\left(\frac{\omega}{\omega_{W}} \sqrt{\frac{1+\beta}{1-\beta}}\right)\right],
\end{aligned}
$$

где

$$
f(x)=\sum_{n=1}^{\infty} \frac{1}{n^{2}}(1+n x) \exp (-n x),
$$

$\omega_{W}=k_{B} T_{1} / \hbar-$ частота Вина.

Заметим, что угловая зависимость (46) характерна для интенсивности излучения любой движущейся системы с изотропным распределением излучаемых квантов в системе покоя тела [15].

На рис. 2 показан вид спектра (47) в приведенных единицах при различных значениях $\beta$. Форма спектра остается близкой к планковской, но частота максимума становится равной $\omega_{\max }=2.82 \gamma \omega_{W}$. В заключение заметим, что все выше рассмотренные характеристики, связанные с динамикой и тепловым излучением АЧТ с радиусами $R \gg \lambda_{W}$, значительно отличаются от таковых для микрочастиц частиц с радиусами $R \ll \lambda_{W}$, не являющихся абсолютно черными $[11,13]$, для которых приближение геометрической оптики $\left(R \gg \lambda_{W}\right)$ неприменимо.

\section{Заключение}

Получены замкнутые выражения для динамических и излучательных характеристик АЧТ с размерами, превышающими виновскую длину волны, имеющего собственную температуру $T_{1}$, и движущегося со скоростью $V$ в 
равновесном газе фотонов с температурой $T_{2}$ : тангенциальной тормозящей силы, интенсивности теплового излучения и поглощения, скорости нагрева (охлаждения), спектрально-угловой интенсивности излучения. Все указанные величины отнесены либо к собственной системе покоя тела, либо к системе отсчета фотонного газа. Показано, что в начальный момент времени суммарная интенсивность излучения может зависеть от формы тела, как, например, для сферических и дискообразных частиц одинакового радиуса. При неизменной температуре $T_{2}$ фонового излучения по прошествии некоторого времени устанавливается квазистационарное тепловое состояние тела с эффективной температурой, зависящей от скорости и $T_{2}$, при котором интенсивность теплового излучения не зависит от формы, а энергия поступательного движения превращается в излучение до момента остановки. Характерное время установления квазистационарного состояния значительно меньше времени торможения, поэтому кинетика теплообмена может рассматриваться при фиксированной скорости движения, а динамика торможения и квазистационарного излучения - при фиксированной температуре тела. Форма спектра излучения в системе отсчета фотонного газа близка к планковской, но максимум смещается в сторону высоких частот пропорционально гаммафактору. Характерные параметры, связанные с излучением и динамикой АЧТ большого радиуса, значительно отличаются от таковых для более мелких (не абсолютно черных) частиц с размерами, меньшими виновской длины волны. Полученные результаты могут быть, в частности, полезны при исследовании эволюции и низкочастотного излучения астероидных тел и планетезималей, движущихся в темных галактических и межгалактических областях космического пространства, слабо освещаемых звездами. Возможно, в будущем результаты будут востребованы при исследовании динамики и электромагнитного излучения частиц в вакуумных ловушках с фиксированной температурой стенок и динамического эффекта Казимира.

\section{Конфликт интересов}

Авторы заявляют, что у них нет конфликта интересов.

\section{Список литературы}

[1] A.W. Rodriguez, P.C. Hui, D.P. Woolf, S.G. Johnson, M. Lonar, F. Capasso. Ann. Phys., 527 (1-2), 45 (2015). DOI 10.1002/andp.201400160

[2] A. Pontin, M. Bonaldi, A. Borrielli, F. Marino, L. Marconi, A. Bagolini, G. Pandraud, E. Serra, G.A. Prodi, F. Marin. Ann. Phys. (Berlin), 527, 89 (2015). https://doi.org/10.1002/andp.201400093

[3] M. Aspelmeyer, T.J. Kippenberg, M. Marquardt. Rev. Mod. Phys., 86, 1391 (2014). https://doi.org/10.1103/RevModPhys.86.1391

[4] R. Reimann, M. Doderer, E. Heberstreit, R. Diehl, M. Frimmer, D. Windey, F. Tebbenjohanns, L. Novotny. Phys. Rev. Lett., 121 (1-5), 033602 (2018).

https://doi.org/10.1103/PhysRevLett.121.033602
[5] J. Ahn, Z. Xu, J. Bang, Yu-Hao Deng, T.M. Hoang, Q. Han, Ren-Min Ma, T. Li. Phys. Rev. Lett., 121 (1-5), 033603 (2018). https://doi.org/10.1103/PhysRevLett.121.033603

[6] D.J. Scheeres. Nature, 512, 139 (2014). https://doi.org/10.1038/512139a

[7] B. Rozitis, E. MacLennan, J.P. Emery. Nature, 512, 174 (2014). https://doi.org/10.1038/nature13632

[8] Y.I. Izotov, N.G. Guseva, K.J. Fricke, E. Krugel, C. Henkel. Astron. Astrophys., 570, 97 (2014). https://doi.org/10.1051/0004-6361/201423539

[9] Thiem Hoang, Ap. J., 876 (1-10), 13 (2019). DOI: $10.3847 / 1538-4357 / a b 1075$

[10] Г.В. Дедков, А.А. Кясов. УФН, 187 (6), 599 (2017). https://doi.org/10.3367/UFNr.2016.12.038006 [G.V. Dedkov, A.A. Kyasov. Physics-Uspekhi, 60 (6), (2017).]

[11] G.V. Dedkov, A.A. Kyasov. J. Appl. Phys. Sci. Int., 9 (4), 124 (2017).

[12] A.A. Kyasov, G.V. Dedkov. Phys. J. (AIS), 2 (3), 176 (2016).

[13] G.V. Dedkov, A.A. Kyasov. Phys. Scripta, 89 (1-7), 105501 (2014). https://doi.org/10.1088/0031-8949/89/10/105501

[14] G.V. Dedkov, A.A. Kyasov. Int. J. Mod. Phys. B, 29 (32), 1550237 (1-9) (2015). DOI: 10.1142/S0217979215502379

[15] Л.Д. Ландау, Е.М. Лифшиц. Теория поля (Наука, М., 1988). [L.D. Landau, E.M. Lifshitz. The Classical Theory of Fields (Pergamon, 1975)] https://doi.org/10.1016/C2009-0-14608-1

[16] G.R. Henry, R.B. Feduniak, J.E. Silver, M.A. Peterson. Phys. Rev., 176 (5), 1451 (1968). http://dx.doi.org/10.1103/PhysRev.176.1451

[17] A.P. Lightman, W.H. Press, R.H. Price, S.A. Teukolsky. Problem Book in Relativity and Gravitation (Princeton Univ. Press, Ney Jersey, 1975)

[18] Физчческие величины. Справочник под ред. И.С. Григорьева, Е.3. Мейлихова. (Энергоатомиздат, М., 1991)

[19] Л.Д. Ландау, Е.М. Лифшиц. Статистическая фбизика. Ч. 1. (Физматлит, M., 2001) [L.D. Landau, E.M. Lifshitz. Statistical Physics. (Pergamon Press, Oxford, 1980), v. 1. 